\title{
Psychophysiological responding to emotional memories in healthy young men after cortisol and propranolol administration
}

\author{
Marieke S. Tollenaar • Bernet M. Elzinga • \\ Philip Spinhoven • Walter Everaerd
}

Received: 19 September 2008 / Accepted: 26 November 2008 / Published online: 13 January 2009

(C) The Author(s) 2009. This article is published with open access at Springerlink.com

\begin{abstract}
Rationale Propranolol is found to reduce physiological hyper-responsiveness in post traumatic stress disorder (PTSD), possibly by affecting reconsolidation after the reactivation of traumatic memories. Cortisol is found to attenuate declarative memory retrieval, but it is unknown whether it also reduces physiological responses to emotional memories.

Objectives To examine whether the effects of propranolol on physiological responding to emotional memories can also be found in healthy controls and to investigate the immediate and prolonged effects of cortisol on physiological responding to emotional memories, we tested these effects in 79 healthy young men.

Materials and methods After preparing a script of a negative disturbing memory, participants were instructed to imagine this event 1 week later after ingestion of either $35 \mathrm{mg}$ cortisol, $80 \mathrm{mg}$ propranolol, or a placebo. Physiological responding to the script-driven imagery was recorded. Another week later, after washout, the imagery was repeated again. During all three sessions as well as 8 months later, subjective emotional reactions to the memories were assessed.
\end{abstract}

M. S. Tollenaar $(\bowtie) \cdot$ B. M. Elzinga $\cdot$ P. Spinhoven

Clinical, Health and Neuropsychology Unit,

Institute for Psychological Research, Leiden University,

P.O. Box 9555, 2300 RB Leiden, The Netherlands

e-mail: mtollenaar@fsw.leidenuniv.nl

P. Spinhoven

Department of Psychiatry, Leiden University,

P.O. Box 9600, 2300 RC Leiden, The Netherlands

W. Everaerd

Department of Clinical Psychology, University of Amsterdam,

Roetersstraat 15 ,

1018 WB Amsterdam, The Netherlands
Results The emotionality of the memories was reduced over time, which was not affected by the treatments, however. The personal emotional script did evoke higher skin conductance responses than a neutral story, which decreased 1 week later, but no effects were found of either propranolol or cortisol on this responsiveness.

Conclusions Whereas healthy males do show psychophysiological responding to personal emotional scripts, the effects of cortisol and propranolol on physiological responses to emotional memories might be specific to clinical groups characterized by hyper-responsiveness, like PTSD. Future studies using longer-acting doses and more elaborate reactivation procedures in both healthy men and women could shed more light on the effects of cortisol and propranolol on psychophysiological responding to emotional memories.

Keywords Memory $\cdot$ Reactivation $\cdot$ Reconsolidation . Cortisol $\cdot$ Propranolol $\cdot$ Physiology $\cdot$ Heart rate .

Skin conductance $\cdot$ PTSD $\cdot$ Phobia

Stress hormones like cortisol and (nor)adrenaline have been found to affect human memory processing (Cahill et al. 1994; Lupien and McEwen 1997; Wolf 2008). These effects are dependent on several variables, including the stage of memory processing and the emotionality of the memories involved. Encoding and consolidation stages seem to be enhanced by both elevated cortisol and adrenaline levels (Andreano and Cahill 2006; Buchanan and Lovallo 2001; Cahill and Alkire 2003; O'Carroll et al. 1999), while they are impaired after blocking adrenaline by means of selective beta-blocking agents like propranolol (Cahill et al. 1994; van Stegeren et al. 1998). On the other hand, retrieval seems to be impaired by increased cortisol levels (de Quervain et al. 
2000; Het et al. 2005), while not much is known about the effects of adrenaline manipulation before retrieval (Chamberlain et al. 2006; de Quervain et al. 2007). Regardless of the memory stage, the effects of cortisol seem to be dependent on the emotionality of the memories involved. That is, effects are stronger when memories are arousing (Buchanan and Lovallo 2001; Kuhlmann et al. 2005a, b) or when the testing environment elicits enough arousal (Abercrombie et al. 2005; Kuhlmann and Wolf 2006; Tollenaar et al. 2008a). Likewise, blocking adrenergic activation impairs the encoding mostly of emotional material (Cahill et al. 1994; van Stegeren et al. 1998).

As it has been shown that encoding and retrieval can be affected by stress hormones, lately, an increasing interest in manipulating postretrieval processes has arisen (e.g., Diergaarde et al. 2008; McCleery and Harvey 2004). If it would be possible to affect memory traces after they have been formed and retrieved, this could improve the treatment of stress- and memory-related disorders, like post traumatic stress disorder (PTSD) and phobias (de Quervain and Margraf 2008; Debiec and Ledoux 2006). Promising in this view is animal research that has shown that stress hormones like corticosterone (a glucocorticoid that resembles cortisol, but is naturally more abundantly present in rodents) and beta-adrenergic blocking agents like propranolol can affect long-term memory when administered during or after reactivation of the existing memory traces (Abrari et al. 2008; Cai et al. 2006; Debiec and Ledoux 2004; Maroun and Akirav 2007; Przybyslawski et al. 1999; Tronel and Alberini 2007; Yang et al. 2005). Processes that are thought to be influenced by these drugs are postretrieval mechanisms like extinction and reconsolidation (Suzuki et al. 2004). While extinction may lead to new memories that are formed during habituation to emotional memories (or conditioned anxiety responses), reconsolidation is thought to be a process during which the original memory trace becomes temporarily labile after reactivation, and thus prone to change. If extinction could be enhanced or reconsolidation impaired, it should be possible to attenuate existing (traumatic and anxious) memories.

Based on the above findings, both cortisol and propranolol have been proposed to lead to lasting reductions of emotional memory traces after exposure to traumatic memories and phobias. Moreover, both substances have already been included in clinical trials. Preliminary results have indeed shown that administration of both cortisol and propranolol can diminish PTSD and anxiety symptoms. Namely, perioperative cortisol administration reduced PTSD symptoms at 6 months after cardiac surgery (Schelling et al. 2004) and repeated cortisol administration was found to reduce symptoms of re-experiencing and intensity of the traumatic memories in PTSD patients (Aerni et al. 2004) and also to reduce phobic fears (Soravia et al. 2006). Propranolol administered within hours of a traumatic experience was found to reduce subsequent physiologic responding to traumatic memories (Pitman et al. 2002) and development of PTSD symptoms (Vaiva et al. 2003). Although these studies show clinically relevant effects of cortisol and propranolol, the mechanisms through which these substances work are still unclear.

During reactivation, two different memory routes could be affected that are not mutually exclusive: (1) declarative memory traces might be weakened and (2) the physiologically arousing components of emotional memories might be attenuated. While the first route is thought to be mostly mediated by the hippocampus and prefrontal cortex, the amygdala is thought to be engaged in the emotional reactions to memories, but these systems are highly interlinked (e.g., Greenberg et al. 2005). Therefore, it is also of interest to know whether these routes can be affected separately. In order to test the first possibility, we previously studied the immediate and prolonged effects of both cortisol and propranolol administration on declarative memory retrieval. We found that declarative memory can be impaired long-term when memories are reactivated during high levels of stress (Tollenaar et al. 2008b) or after cortisol administration (Tollenaar et al. 2008c), in line with animal research (Cai et al. 2006; Maroun and Akirav 2007). In contrast, we did not find any immediate or long-term effects of propranolol on declarative memory after reactivation (Tollenaar et al. 2008c), which is consistent with findings by de Quervain et al. (2007).

In line with the idea that the physiologically arousing components of emotional memories can be attenuated, a recent study by Brunet et al. (2008) has shown that postretrieval propranolol administration diminished physiological responses to script-driven imagery of traumatic memories in PTSD patients. These results might indicate that propranolol is more effective in attenuating emotional components of memories than reducing declarative memory. Het and Wolf (2007) found that cortisol administration in healthy young women led to reduced negative mood after a psychosocial stress task. They suggest that this effect might be mediated by a slight impairment in retrieving the just-experienced negative stress episode and/or from a reduced retrieval of previous negative episodes related to the stressor. This finding indicates that cortisol administration might affect the emotional experience of negative events and thus possibly also of negative memories.

To investigate whether the physiologically reducing effects of propranolol in PTSD patients can also be found in a healthy human population and to examine whether cortisol has similar properties of attenuating the physiological components of emotional memories, we have conducted the present study. In the present study, we investigated the immediate and prolonged effects of both 
cortisol and propranolol administration on physiological responding to script-driven imagery of negative, disturbing memories in healthy young men after reactivation of these memories, as well as on subjectively experienced emotions to these memories. We expected both propranolol and cortisol to influence postretrieval processes leading to diminished physiological responding to the emotional script in comparison to a neutral story 1 week after treatment.

\section{Materials and methods}

\section{Participants}

Eighty-five Dutch male students were recruited through advertisements at colleges and the Leiden University as part of a larger study on memory for which results will be presented elsewhere (Tollenaar et al. 2008c). Only men were selected because of possible confounding effects of menstrual cycle and contraceptive pills on the relation of cortisol and propranolol treatment with memory (Cahill and van Stegeren 2003; Kuhlmann and Wolf 2005). Participants were screened before inclusion. Inclusion criteria were: no reported history of disease or psychiatric problems, no current use of prescribed medication including corticosteroid-containing ointments, no chronic disease requiring medical attention including diabetes, allergies, and asthma, no use of psychotropic drugs, no alcohol abuse, smoking less than ten cigarettes per day, age between 18 and 35 years, an estimated body mass index (BMI) between 19 and $26 \mathrm{~kg} / \mathrm{m}^{2}$, and blood pressure levels over 100/ $70 \mathrm{mmHg}$. Before participation, written informed consent was obtained, and after participation, participants were rewarded with either course credits or a monetary compensation (40 Euros). The study protocol was approved by the Medical Ethics Committee of the Leiden University Medical Center.

To minimize influences on baseline cortisol levels, participants were instructed to refrain from drinking any sweet or caffeinated drinks and eating heavy meals on the morning of the second (treatment) session. Furthermore, they were instructed not to eat or drink anything but water and not to smoke an hour before the second session would start.

Of the 85 recruited participants, two men were excluded after the first session due to low blood pressure. Two participants were ill during one of the sessions and one person dropped out after the first session. We excluded one more participant due to a fire alarm on the second session during the imagery task. Hence, 79 participants completed the study. Participants were randomly assigned to one of three experimental groups in a double-blind betweensubjects design (placebo: $N=26$, cortisol: $N=26$, propranolol: $N=27)$. Dependent on group, $35 \mathrm{mg}$ hydrocortisone, $80 \mathrm{mg}$ fast-acting propranolol, or a placebo was administered orally, in identical capsules.

Table 1 shows the demographic variables of the participants per group. No differences between groups were found for BMI, anxiety (STAI-trait), and general psychopathology (Symptoms Checklist-90 [SCL-90]). Age was significantly higher in the cortisol group compared to the placebo group $(t(32)=2.38, p<0.05)$ due to the fact that the two oldest participants (aged 30 and 32 years) were randomly assigned to the cortisol group. Depression scores on the Beck Depression Inventory (BDI-II) were marginally higher in the control group compared to both the cortisol $(t(44)=1.74$, $p=0.09)$ and propranolol group $(t(50)=1.89, p=0.07)$.

\section{Psychophysiological measures}

Saliva samples were obtained using Salivettes (Sarstedt, Germany) to measure unbound cortisol and alpha-amylase levels. Alpha-amylase has been shown to be an estimate of adrenergic activity (Nater et al. 2006; Rohleder et al. 2004) and is sensitive to beta-blockage by propranolol (van Stegeren et al. 2005). Saliva samples were stored at $-20^{\circ} \mathrm{C}$ prior to analyses. The saliva samples were analyzed by the Kirschbaum Lab, Technical University of Dresden (see Rohleder et al. 2006). One person (from the propranolol group) had a missing saliva sample and three people (one from each group) had a missing alpha-amylase sample.

Table 1 Demographic variables (mean \pm SD)

\begin{tabular}{lccc}
\hline Group & $\begin{array}{l}\text { Placebo } \\
(N=26)\end{array}$ & $\begin{array}{l}\text { Cortisol } \\
(N=26)\end{array}$ & $\begin{array}{l}\text { Propranolol } \\
(N=27)\end{array}$ \\
\hline Age & $19.54(1.39)^{*}$ & $21.35(3.61)^{*}$ & $20.74(2.21)$ \\
BMI & $22.13(2.38)$ & $22.40(1.98)$ & $21.90(2.31)$ \\
Depression (BDI-II) & $6.54(4.46)^{* *}$ & $4.69(3.04)^{* *}$ & $4.46(3.41)^{* *}$ \\
Anxiety (STAI-trait) & $33.81(9.26)$ & $33.73(9.08)$ & $31.56(6.89)$ \\
Psychopathology (SCL-90) & $28.50(25.26)$ & $28.73(23.23)$ & $27.81(19.88)$ \\
\hline
\end{tabular}

$B M I$ body mass index, BDI-II Beck Depression Inventory II, SCL-90 Symptoms Checklist-90

${ }^{*} p<0.05$, significant difference in age between the placebo and cortisol groups; $* * p<0.10$, marginally significant difference in depression scores between the placebo, cortisol, and propranolol groups 
These participants were left out of the repeated-measures analysis of variance (RM-ANOVA) with cortisol or alphaamylase as a factor.

Heart rate and blood pressure were measured with an automatic upper arm blood pressure monitor (OMRON, M6) once before $(t=0)$ and three times after pill ingestion (at $t=75,110$, and $135 \mathrm{~min}$ ) to further asses adrenergic functioning. In addition to each physiological recording, participants were given a questionnaire with seven questions on the intensity of subjective experiences of tension, anxiety, insecurity, irritation, motivation, mood, and tiredness. Answers were given on visual analog scales (VAS) of $100 \mathrm{~mm}$ in length, leading to a score from 0 (not at all) to 100 (extremely) on each scale.

During the script-driven imagery procedures, heart rate and skin conductance level (SCL) were continuously measured at $50 \mathrm{~Hz}$ using a stimulus presentation and physiological analyses software package developed by the University of Amsterdam (VSRRP98; http://www.test.uva. nl/ozi_psychology/index.php?Page=Software). Heart rate was measured with a finger plethysmograph on the nondominant ring finger. SCL was measured with two $1-\mathrm{cm}^{2}$ electrodes attached to the middle phalanx of the index and middle finger of the same hand. SCL fluctuations (SCLfluc) were calculated in Matlab (R2007a) by peak detection on the first derivative of the SCLs after a second-order forward/backward $1 \mathrm{~Hz}$ low pass filter. Because statistical analyses on the SCLfluc responses showed similar patterns to SCL responses, we will not report the SCLflucs in the "Results" section. For nine participants, heart rate was not measured on one or more time points due to technical failure (placebo: $N=5$, cortisol: $N=4$ ). These participants were left out of the RM-ANOVAs with heart rate responding as a factor.

\section{Questionnaires}

The BDI-II (Beck et al. 1996; van der Does 2002) was administered to assess depressive feelings in the past 2 weeks, a Dutch version of the STAI-trait (Spielberger 1983) to measure the level of generalized anxiety, and the SCL-90
(Arrindell and Ettema 1986) to assess psychological symptoms and general psychopathology during the last week.

A questionnaire about the personal script consisted of questions on emotional arousal, valence, re-experiencing, fear, anger, sadness, importance, and how often one had thought about the event. All were measured on seven-point Likert scales, ranging from 1 (very low) to 7 (very high).

\section{Procedure}

Participants came to a laboratory at the Faculty of Social and Behavioral Sciences in Leiden for three sessions. The interval between each session was 1 week (see Fig. 1 for an overview of the test sessions). In the first session, screening measurements of blood pressure and heart rate were taken with the OMRON after three rest periods of $4 \mathrm{~min}$, as well as a baseline measure of heart rate and SCL during a 4-min continuous measurement period. During this first session, a personalized script was prepared in 15 min (according to methodology of Bremner et al. 1999; Pitman et al. 1987). Participants were asked to write down a negative disturbing event that still triggered emotional feelings of anxiety, anger, or fear on a script preparation form in the present tense. Participants also filled in a short questionnaire on the intensity of emotions the memory evoked. After that session, before session 2, the experimenter reviewed the writing and composed and recorded a script approximately $1 \mathrm{~min}$ in length for later audio playback. At the start of the second session, baseline measurements of heart rate and blood pressure were assessed and baseline saliva samples obtained. Participant then ingested a capsule containing placebo, $35 \mathrm{mg}$ hydrocortisone, or $80 \mathrm{mg}$ propranolol. During the next $75 \mathrm{~min}$, participants completed several computer-based questionnaires and were instructed to remain in the laboratory and read (reading material was provided). At $t=75 \mathrm{~min}$ after ingestion, participants heart rate and blood pressure were again assessed and saliva measurements obtained. At approximately $t=90$, the scriptdriven imagery task took place. After a baseline period of $60 \mathrm{~s}$, a neutral script, which was similar for all participants, was played with the VSRRP software program while
Fig. 1 Schematic overview of the test sessions

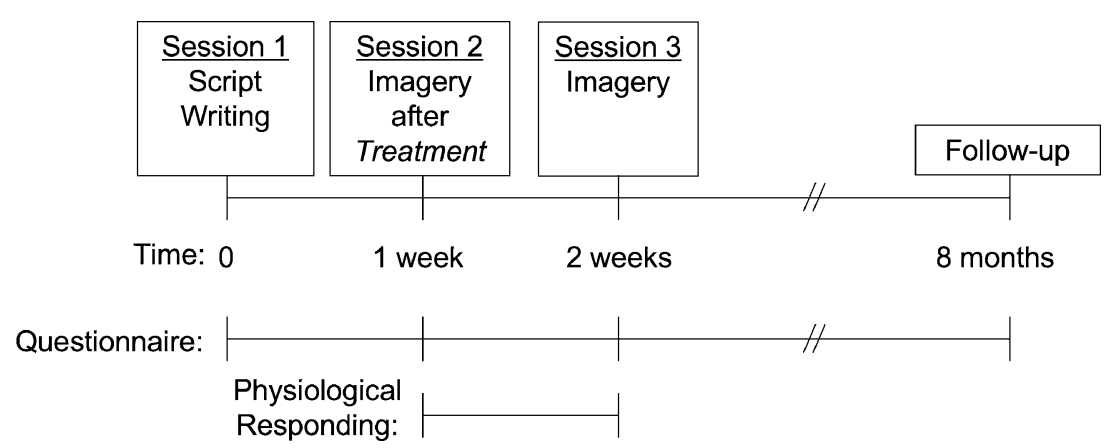


physiological reactions were measured. Then, for $60 \mathrm{~s}$, participants were instructed to imagine the story they had heard. After another baseline period of $60 \mathrm{~s}$, their personal script was played, followed by a 60 -s period in which they had to imagine reliving the event. After the imagery task, participant filled in the emotionality questionnaire again. The neutral story was always followed by the personal script to prevent emotions elicited by the personal scripts from persisting into the neutral story. In the third session, the same procedures regarding the imagery tasks were repeated, also followed by the emotionality questionnaire. In an 8-month follow-up telephone interview, participants were asked once more to rate the intensity of the emotions related to their personal memory.

Data analysis

The effects of the treatment (placebo versus cortisol or propranolol) on physiological and subjective measures were analyzed using RM-ANOVAs with time as within-subject and group as between-subject factors. Log-transformed values were used for cortisol and alpha-amylase values to account for non-normality. The change in subjective emotionality of the story over time was analyzed using an repeated-measures multivariate analysis of variance (RMMANOVA) with group as a between-subject factor and time (session 1, session 2, session 3, and 8 months followup) as a within-subject factor. For the analyses of the physiological responding to the script-driven imagery, mean SCL and heart rate were calculated for 60 -s periods by averaging measurements of three consecutive 20 -s periods. Reactions to the neutral and personal script were calculated by subtracting the 1-min listening and 1-min imagery periods from the 1-min baseline period before the respective story. This resulted in two neutral change scores and two emotional script change scores on both sessions 2 and 3 for each group. These change scores were square roottransformed prior to analyses. We applied RM-ANOVAs with group as a between-subject factor and session (session 2 versus session 3), emotion (neutral versus personal script), and part (listening versus imagining) as withinsubject factors. In a multivariate RM analysis, we included heart rate and SCL change scores as dependent variables. Then, separate RM-ANOVAs were calculated for each dependent change score separately. Greenhouse-Geisser corrected $p$ values were used when indicated by violated sphericity. Analyses were performed with SPSS 14.0 (SPSS, Chicago, IL, USA). The criterion for statistical significance was $p<0.05$.

\section{Results}

Treatment effects

Cortisol administration induced the expected increase in free saliva cortisol levels, as indicated by a significant time by group interaction between the cortisol and placebo groups $[F$ $(2,112)=345.96, p<0.001$; see Table 2]. Cortisol did not affect alpha-amylase levels, heart rate, and systolic (SBP) or diastolic (DBP) blood pressure $[F(2,93)=2.34, p=0.10 ; F(2$, $87)=1.86, p=0.71 ; F(2,113)=0.56, p=0.59 ; F(2,114)=$ $1.71, p=0.18$, respectively], see also Fig. 2a-d.

Propranolol lowered adrenergic activation as expected, indicated by significant time by group interactions between the propranolol and placebo groups for alpha-amylase, heart rate, and $\operatorname{SBP}[F(2,111)=3.88, p<0.02 ; F(2,101)=16.25$, $p<0.001 ; F(2,101)=10.76, p<0.001$, respectively]. All measures declined stronger over time in the propranolol group compared to the placebo group (see Fig. 2a-d). The general decline in adrenergic activation in all groups from $t=0$ to $t=75$ might be due to the 75 -min restful waiting period. Although DBP also showed a time by group interaction $[F(2,125)=4.15, p<0.02]$, post hoc $t$ test revealed no significantly lower DBP at any of the time points (all $p \mathrm{~s}>0.09)$. Propranolol also slightly increased free saliva cortisol over time compared to the control group $[F(2,94)=$ 12.74, $p<0.001]$ (see Tollenaar et al. 2008c).

No effects of treatment over time were found on subjective feelings of tension, anxiety, insecurity, irritation, motivation, mood, and tiredness (all $p \mathrm{~s}>0.39$ ). We did find an interaction effect of group with time on anxiety, although not significant $[F(5,186)=2.21, p=0.06]$, suggesting that the propranolol group reported lower anxious

Table 2 Free salivary cortisol in nanomoles per liter $( \pm$ SEM $)$ in each treatment group

\begin{tabular}{llrrr}
\hline & \multicolumn{1}{l}{ Time } & & & \\
\cline { 2 - 5 } Group & $t=0$ & $t=75$ & $t=110$ & $t=135$ \\
\hline Placebo & $9.11(0.96)$ & $5.00(0.45)$ & $4.34(0.39)$ & $4.58(0.44)$ \\
Cortisol & $7.47(0.71)$ & $206.61(17.53)^{*}$ & $134.79(9.01)^{*}$ & $99.37(5.55)^{*}$ \\
Propranolol & $8.01(0.47)$ & $6.38(0.78)$ & $8.29(1.05)^{* *}$ & $9.67(1.27)^{* *}$ \\
\hline
\end{tabular}

${ }^{*} p<0.001$, significant increase in cortisol levels in the cortisol group versus the placebo group; ${ }^{*} p<0.001$, significant increase in cortisol levels in the propranolol group versus the placebo group 
Fig. 2 Physiological measures (mean \pm SEM) of adrenergic activation on session 2 before treatment $(t=0)$ and after treatment $(t=75,110$, and $135 \mathrm{~min})$ : a alpha-amylase, $\mathbf{b}$ heart rate, $\mathbf{c}$ SBP, d DBP. U/L units per liter, bpm beats per minute, $\mathrm{mmHg}$ millimeter of mercury. ${ }^{*} p<0.01$ significant difference in the propranolol versus the placebo group; $\# p<0.05$, significant difference in the propranolol versus the placebo group

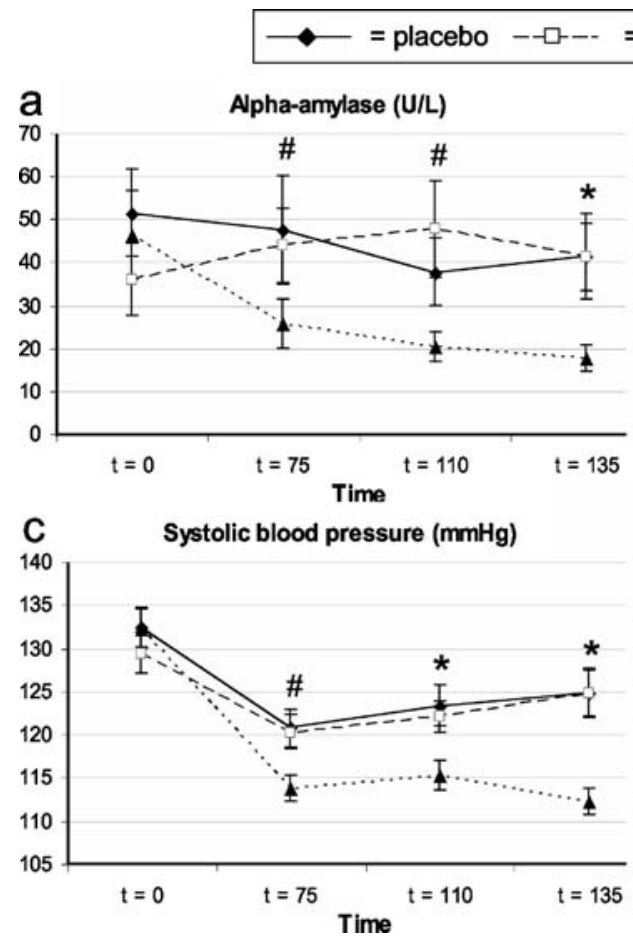

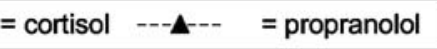
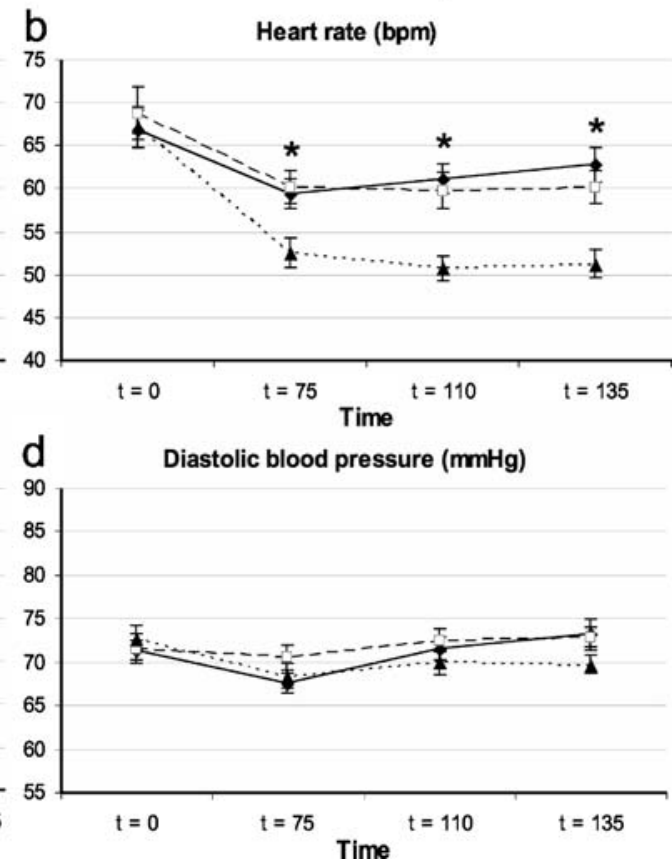

feelings over time compared to the control group. However, post hoc $t$ tests revealed no significantly lower anxious feelings at any of the time points in the propranolol group versus the control group (all $p s>0.11$ ). Furthermore, all treatments were well-tolerated and participants were not aware which treatment they received (Pearson $\chi^{2}(6)=5.71$, $p=0.46$ )

Baseline values

In the first session, a baseline period was measured for heart rate and SCL. No differences were found between the groups (MANOVA: $F(6,148)=0.67, p=0.61$; separate $p s>0.34$; see
Table 3). The groups also did not differ on any of the emotionality ratings that were given to their personal script on the first session (MANOVA: $F(16,140)=0.47, p=0.96$; all separate $p s>0.16$; see Table 3 ).

Emotional ratings over time

To study the change over time in the subjective emotionality ratings related to the personal script, we performed a RMMANOVA with time (session 1, session 2, session 3, and follow-up) as a within-subject factor and group as a betweensubject factor. Follow-up was completed by 74 participants. The overall MANOVA revealed no significant group by time

Table 3 Mean $( \pm \mathrm{SD})$ physiological and subjective baseline values on session 1 for the three treatment groups

\begin{tabular}{|c|c|c|c|}
\hline \multirow[b]{2}{*}{ Baseline value (session 1) } & \multicolumn{3}{|l|}{ Group $^{a}$} \\
\hline & Placebo & Cortisol & Propranolol \\
\hline Heart rate (bpm) & $65.85(8.27)$ & $64.33(8.61)$ & $65.17(9.54)$ \\
\hline $\mathrm{SCL}(\mu \mathrm{S})$ & $16.91(5.54)$ & $18.42(6.16)$ & $16.18(6.13)$ \\
\hline Arousal & $4.04(1.54)$ & $4.54(1.61)$ & $4.33(1.82)$ \\
\hline Negative valence & $4.81(1.47)$ & $4.96(1.56)$ & $5.15(1.32)$ \\
\hline Re-experiencing & $4.19(1.47)$ & $4.31(1.35)$ & $4.26(1.38)$ \\
\hline Fear & $2.54(1.27)$ & $2.81(1.58)$ & $2.85(1.46)$ \\
\hline Anger & $2.77(1.68)$ & $3.42(1.65)$ & $3.37(1.84)$ \\
\hline Sadness & $3.77(1.77)$ & $4.08(1.85)$ & $4.41(1.62)$ \\
\hline Importance & $3.65(1.92)$ & $4.27(1.89)$ & $4.63(1.74)$ \\
\hline Thought about & $4.19(1.55)$ & $4.54(1.68)$ & $4.56(1.34)$ \\
\hline
\end{tabular}

On the subjective emotionality ratings, minimum scores were 1 (very low) and maximum scores were 7 (very high)

$S C L$ skin conductance level, $\mathrm{bpm}$ beats per minute, $\mu S$ microsiemens

${ }^{\mathrm{a}}$ No effects of group on any of the baseline measures were found (all $p \mathrm{~s}>0.16$ ) 
interactions $[F(42,1,272)=0.72, p=0.91]$, but significant declines over time $[F(21,627)=5.43, p<0.001]$ reflected in significant univariate ANOVA tests for all measures (all $p \mathrm{~s}<0.001$, except for fear $p<0.02$ ). The decline in subjective emotional appraisals was already significant at session 3 $[F(14,302)=3.90, p<0.001]$.

\section{Physiological responses to the scripts}

Figure 3 shows the raw heart rate and SCL responses to both the neutral and personal emotional script in the three groups. For every session, responses were divided into the listening and imagery responses. The RM-MANOVA on heart rate and SCL showed a main effect of emotion $[F(2$, $66)=24.38, p<0.001]$, a main effect of day $[F(2,66)=4.84$, $p<0.02]$, a main effect of part $[F(2,66)=36.36, p<0.001]$, and a day by emotion interaction $[F(2,66)=5.08, p<0.01)$, as well as a part by day $[F(2,66)=3.52, p<0.05]$ and a part by emotion interaction $[F(2,66)=5.64, p<0.01]$. No main or interaction effects of group were found (all $p s>0.10$ ), thus not revealing the expected emotion by group or day by emotion by group interactions.

The separate RM-ANOVA for SCL responses revealed the same main and interaction effects as in the RM-MANOVA [days: $F(1,76)=8.99, p<0.01$; emotion: $F(1,76)=53.01$, $p<0.001$; part: $F(1,76)=92.95, p<0.001$; day by emotion: $F(1,76)=7.56, p<0.01)$; part by day $(F(1,76)=9.43$, $p<0.01)$; and part by emotion interaction: $F(1,76)=18.46$, $p<0.001]$. Overall, the emotional script seemed to elicit higher SCL responses than the neutral story, and on the third session, responses were smaller than on the second session. To examine the day by emotion interaction, we split the analyses by emotion. It was revealed that there was no day effect for the neutral story $[F(1,76)=0.033, p=0.86]$, but a significant day effect for the emotional script $[F(1,76)=$ $13.98, p<0.001]$ with lower SCLs to the emotional script on the third session compared to the second session (see also Fig. 3c,d). Furthermore, overall responses to the listening part were higher than to the imagery part. To examine the part by day and the part by emotion interactions, we split the analyses by part. During both the listening and imagining part, the main emotion effect was present, although slightly stronger during listening than imagery $[F(1,76)=75.04, p<$ 0.001 and $F(1,76)=31.90, p<0.001$, respectively]. The day by emotion interaction was also present for both parts, but the main effect for day was only present for the imagery part $[F(1,76)=23.30, p<0.001$; listening part: $F(1,76)=1.22, p=$ $0.27]$, reflecting a greater decrease in physiological response over time for imagery.

For heart rate responses, we found no effects of day, emotion, or part (all $p \mathrm{~s}>0.24$ ) as in the RM-MANOVA, but we did find a significant part by emotion by group interaction $[F(2,67)=3.16, p<0.05]$ and a trend for a part by day by group interaction $[F(2,67)=2.54, p=0.09]$. When breaking up the analyses in the listening and imagery part, we found a marginally significant emotion effect only during imagery $[F(1,75)=3.64, p=0.06]$ with higher heart rate change scores for the neutral compared to the emotional story (see Fig. 3a,b). Furthermore, during imagery, we found a trend for a day by group interaction $[F(2,75)=2.84$, $p=0.07]$, revealing that the propranolol group showed higher heart rate responses on session 3 compared to session $2[F(1$, 26) $=6.98, p<0.02]$, while this affect was not present in the control and cortisol groups $(p \mathrm{~s}>0.80)$.
Fig. 3 Heart rate $(\mathbf{a}, \mathbf{b})$ and SCL $(\mathbf{c}, \mathbf{d})$ responses to both the neutral story and personal emotional script in the three groups on both session 2 (after treatment, left) and session 3 (1 week later, right). Responses were divided into listening and imagery responses. $\mathrm{bpm}$ beats per minute, $\mu S$ microsiemens

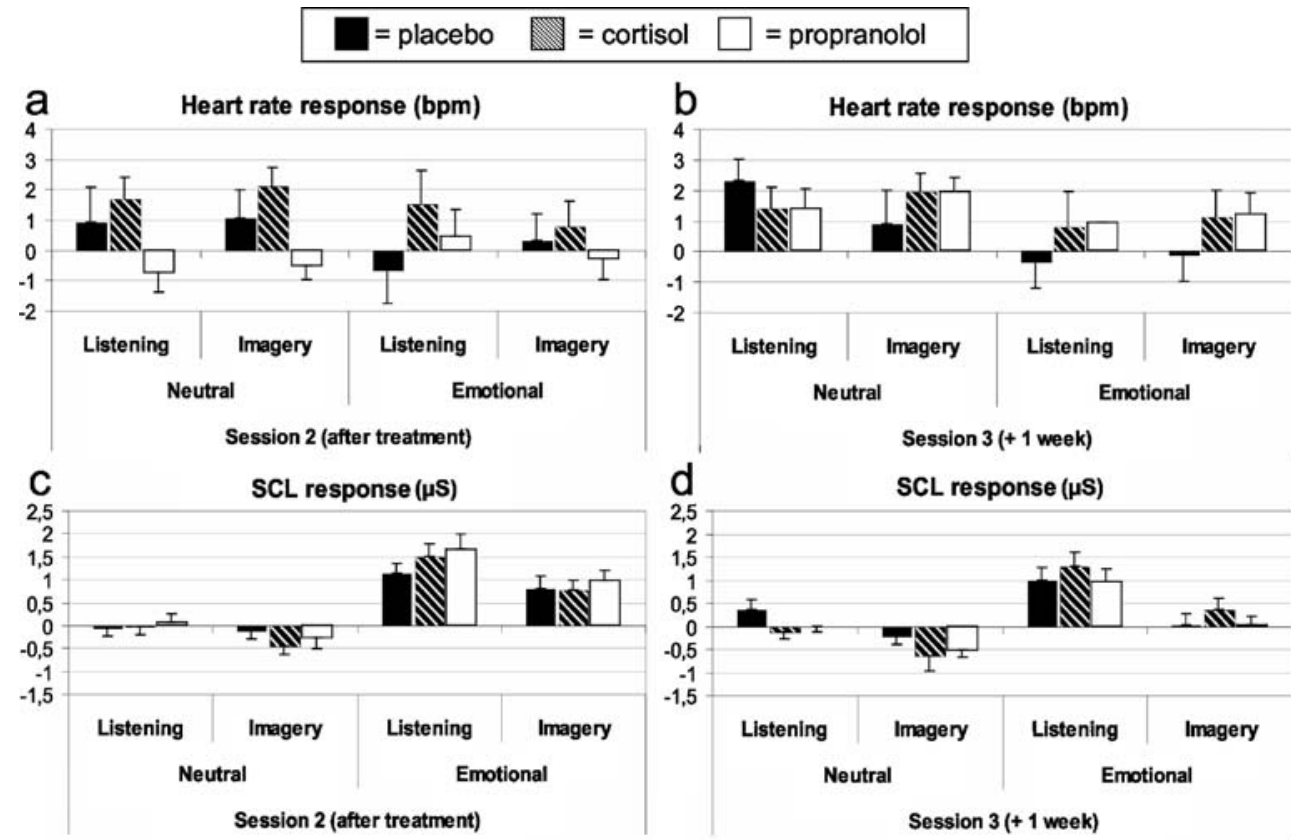


Because differences between groups in age and BDI scores were (borderline) significant, we entered them as covariates in the above analyses. Still none of the expected group effects revealed significance (all $p s>0.29$ ), and the day by group interaction during the imagery part remained borderline significant for heart rate $[F(2,72)=3.01, p=0.06]$.

\section{Discussion}

The present study examined the immediate and prolonged effects of both cortisol and propranolol administration on physiological responses to script-driven imagery of negative, disturbing memories in healthy young men after reactivation of these memories. No diminishing effect of either propranolol or cortisol on psychophysiological responding to the script-driven imagery of emotional memories was found. The subjective emotional experience of the memories was not affected by cortisol or propranolol either. Even though propranolol was found to attenuate physiological responding (heart rate and SCL) to traumatic scripts in PTSD patients (Brunet et al. 2008), we did not find such effects to negative emotional scripts in healthy young men. These results also contrast the finding by Het and Wolf (2007) that cortisol can affect the emotional experience of negative events and the finding that cortisol can reduce the intensity of traumatic memories (Aerni et al. 2004). However, methodological differences between these studies and our study might have caused these conflicting findings, as we will discuss below.

While our hypotheses regarding cortisol and propranolol were not confirmed, we were able to evoke physiological responses to personal emotional scripts in a healthy male population. That is, the emotional scripts led to significantly higher SCL responses than a neutral story, although not to any significant increases in heart rate. Heart rate even seemed to be lower during imagery of the emotional script than during the neutral script. This is in line with previous research showing heart rate decelerations during attention to emotional (auditory) stimuli in healthy humans (Bradley and Lang 2000; Palomba et al. 1997; van Stegeren et al. 2002). Within the propranolol group, heart rate responses to the neutral and emotional script were higher 1 week after treatment than during treatment. This is probably due to the fact that propranolol administration on the second session lowered heart rate and adrenergic functioning overall, and might thus have led to lower heart rate responses in the second session. Therefore, these sessions cannot be compared validly. Overall, the SCL responses were higher when participants listened to their script than when imagining the script, and responses to imaging decreased more over time. This might be important for future studies using script-driven imagery tasks to take into account, since the two processes could be differently affected by drug treatment.

Furthermore, the subjective emotional and arousal responses to the memories decreased steadily over time, from session 1 to session 3 and 8 months later, and likewise the SCL responses to the emotional scripts decreased from the second to the third session. However, while physiological reactions to the emotional memories might have diminished over time, participants might also have been less surprised the second time they heard their story (on the third session). That is, even though subjects knew their memory would be part of the study, on the second session, they were not aware they would hear an audio version of it.

There might be several reasons why cortisol and propranolol did not attenuate physiological responses to script-driven imagery of negative, disturbing memories in healthy young men after reactivation of these memories. First of all, heart rate was not heightened in response to the emotional script in the placebo group on either of the two sessions, and SCLs were not heightened in response to emotional imagery in the placebo group on the last session. Therefore, cortisol and propranolol could only act on SCL responses during the second (treatment) session and on SCL responses to the listening part during the third session, on which we expected the reconsolidation effects. The negative disturbing events that the young, healthy males in our studies had described were probably much less intense than traumatic memories in PTSD populations and hence attenuation of physiological responding might not have been possible due to floor effects. However, we did elicit consistent SCL responses during listening to the emotional scripts and these negative memories were described as overall important and were thought about more often than other memories. This might lead to consideration of a second possibility, namely, that the dose was too low to affect the physiological responding. In the studies by Brunet et al. (2008) and Pitman et al. (2002), multiple doses or longer-acting propranolol were used. These studies involved patient groups that are characterized by high blood pressure and heart rate levels overall, and therefore, larger doses of propranolol might be needed to reduce physiological responding. However, reconsolidation of emotional memories could possibly take longer than a few hours, and longer-acting doses might, therefore, be needed to affect these reconsolidation processes. Cortisol on the other hand, was given in lower doses in the clinical studies by Aerni et al. (2004) and Schelling et al. (2004), but the doses were given daily instead of once, leading to active cortisol during potentially multiple memory reactivations. While in the present study the dose may have been too low or shortacting to obtain reductions in physiological arousal in response to personalized scripts, in the same population as the present study, we did find long-term impairing effects of 
the 35-mg cortisol dose on declarative memory retrieval (Tollenaar et al. 2008c). Taken together, this might lead to the possibility that cortisol only affects declarative memory retrieval and not the physiologically arousing components of the memory. Perhaps longer-acting doses or more frequent administration of cortisol and propranolol might lead to attenuating physiological responding in a healthy population as well.

Our study differed in several aspects to the study in PTSD patients by Brunet et al. (2008). As mentioned, different and longer-acting doses of propranolol were used than in our study. Furthermore, in the study by Brunet et al., propranolol was given after the script preparation procedure that was used to reactivate the event. In our study, the script was prepared a week before drug administration and only the listening to and imagining of the memory, which lasted $2 \mathrm{~min}$, was used to reactivate the memory under the influence of the drugs. This might have been a suboptimal reactivation procedure to affect reconsolidation. In addition, reactivation of the memory a week before treatment might have promoted extinction of or habituation to the memory. Hence, it cannot be ruled out that the reactivation procedure was not optimal to find immediate or prolonged effects of cortisol or propranolol on physiological responding.

Another issue that merits consideration is the timing of the drugs. In the present study, we administered cortisol and propranolol before reactivation. This way both retrieval and postretrieval processes are within the active time window of the drugs. In animal research, these substances are usually given after reactivation to only affect postretrieval processes. In the study by Brunet et al. (2008), a similar postreactivation approach was taken, although in the clinical trials mentioned in the introduction (Aerni et al. 2004; Pitman et al. 2002; Soravia et al. 2006; Vaiva et al. 2003), cortisol and propranolol were administered during a longer time span or before retrieval as well. The fact that, in this study, drugs were active during both retrieval and postretrieval processes could potentially explain our nonresults with regard to the prolonged effects of cortisol and propranolol. That is, if memory retrieval is reduced by cortisol or propranolol, the emotional memories might not be sufficiently reactivated and hence reconsolidation processes could possibly not be blocked. However, psychophysiological responding under the influence of cortisol did not seem to be affected, indicating that the emotional memories were adequately reactivated at the time of the treatment. Propranolol, on the other hand, did lower heart rate responses during treatment, which may have reduced the possibility to affect reconsolidation processes.

A last factor to take into account is that we studied only males. Females might be more reactive to imagery of emotional memories or more sensitive to the effects of cortisol and propranolol, although in the clinical studies no gender effects are reported. Het and Wolf (2007) did find attenuating effects of cortisol on the experience of a negative emotional event in healthy women while we did not find such effects on negative emotional memories in men, suggesting that gender may indeed play an important role. Our negative findings on cortisol and propranolol could also be due to power problems. However, our group sizes compare well to the clinical studies, and as reported above, before we did find an impairing effect of cortisol on declarative memory retrieval in the same population.

We were not able to find an attenuating effect of cortisol on physiological responding to memories in healthy men, but whether cortisol can attenuate physiological responding in PTSD remains unknown. Potentially, the attenuating effects of cortisol are only present in individuals that are hyperaroused. Future studies using cortisol or propranolol in healthy populations could use different or longer-acting doses, more frequent administration, or different timing protocols with regard to reactivation of the memories. Future studies could also investigate more elaborate reactivation paradigms or consider vulnerable populations to elicit higher emotional responses to the memories. In addition, both males and females should be included in future investigations. Conditioning paradigms would also be a good way to measure and replicate animal studies on postretrieval processes. At this point, there are only preliminary data available, showing reducing effects of propranolol on a conditioned fear response in healthy subjects when administered during reactivation of the fear memory (Miller et al. 2004).

To conclude, the present study was able to measure physiological responding to script-driven imagery of emotional memories on two consecutive occasions in healthy young men, reflected in heightened SCL responses and lowered heart rate responses. Furthermore, we measured the subjective emotional responses to these memories over a long time span of 8 months. Reductions in emotional appraisal of the memories were shown within 3 weeks and even further up to 8 months. We did not find any immediate or prolonged effects of either cortisol or propranolol on these physiological and subjective measures. We might conclude that the effect of propranolol on physiological responses to emotional memories is specific to clinical groups characterized by hyper-responsiveness, like PTSD, although differences in study designs might partly explain these divergent findings. Furthermore, the effects of cortisol on physiological responses to emotional memories in clinical groups should still be explored, in addition to its effect on declarative memory retrieval. More knowledge on the mechanisms behind propranolol and cortisol in treating disorders like PTSD and phobias might lead to more efficient and safe use of these drugs (for discussions, see Glannon 2006; van Stegeren 2005). 
Acknowledgements We thank Dagmar Feenstra, Danielle Volker, Laura Wisse, and Liesbeth Kuiper for their help in collecting the data. We also thank Thijs Schrama and Bert Molenkamp for their help in analyzing the physiological data and the anonymous reviewers for their helpful suggestions. This work was supported by the Dutch Organization for Scientific Research, NWO, Grant 400-03-210. The experiments comply with the current laws of The Netherlands.

Open Access This article is distributed under the terms of the Creative Commons Attribution Noncommercial License which permits any noncommercial use, distribution, and reproduction in any medium, provided the original author(s) and source are credited.

\section{References}

Abercrombie HC, Speck NS, Monticelli RM (2005) Endogenous cortisol elevations are related to memory facilitation only in individuals who are emotionally aroused. Psychoneuroendocrinology 31:187-196

Abrari K, Rashidy-Pour A, Semnanian S, Fathollahi Y (2008) Administration of corticosterone after memory reactivation disrupts subsequent retrieval of a contextual conditioned fear memory: dependence upon training intensity. Neurobiol Learn Mem 89:178-184

Aerni A, Traber R, Hock C, Roozendaal B, Schelling G, Papassotiropoulos A, Nitsch RM, Schnyder U, de Quervain DJF (2004) Low-dose cortisol for symptoms of posttraumatic stress disorder. Am J Psychiatry 161:1488-1490

Andreano JM, Cahill L (2006) Glucocorticoid release and memory consolidation in men and women. Psychol Sci 17:466-470

Arrindell WA, Ettema JHM (1986) Handleiding SCL-90 [Manual]. Swets and Zeitlinger, Lisse

Beck AT, Steer RA, Brown GK (1996) Beck Depression Inventory (BDI-II). Manual. The Psychological Corporation, San Antonio, TX, USA

Bradley MM, Lang PJ (2000) Affective reactions to acoustic stimuli. Psychophysiology 37:204-215

Bremner JD, Staib LH, Kaloupek D, Southwick SM, Soufer R, Charney DS (1999) Neural correlates of exposure to traumatic pictures and sound in Vietnam combat veterans with and without posttraumatic stress disorder: a positron emission tomography study. Biol Psychiatry 45:806-816

Brunet A, Orr SP, Tremblay J, Robertson K, Nader K, Pitman RK (2008) Effect of post-retrieval propranolol on psychophysiologic responding during subsequent script-driven traumatic imagery in post-traumatic stress disorder. J Psychiatr Res 42:503506

Buchanan TW, Lovallo WR (2001) Enhanced memory for emotional material following stress-level cortisol treatment in humans. Psychoneuroendocrinology 26:307-317

Cahill L, Alkire MT (2003) Epinephrine enhancement of human memory consolidation: interaction with arousal at encoding. Neurobiol Learn Mem 79:194-198

Cahill L, van Stegeren A (2003) Sex-related impairment of memory for emotional events with beta-adrenergic blockade. Neurobiol Learn Mem 79:81-88

Cahill L, Prins B, Weber M, McGaugh JL (1994) Beta-adrenergic activation and memory for emotional events. Nature 371:702 704

Cai WH, Blundell J, Han J, Greene RW, Powell CM (2006) Postreactivation glucocorticoids impair recall of established fear memory. J Neurosci 26:9560-9566
Chamberlain SR, Muller U, Blackwell AD, Robbins TW, Sahakian BJ (2006) Noradrenergic modulation of working memory and emotional memory in humans. Psychopharmacology (Berl) 188:397-407

de Quervain DJF, Margraf J (2008) Glucocorticoids for the treatment of post-traumatic stress disorder and phobias: a novel therapeutic approach. Eur J Pharmacol 583:365-371

de Quervain DJF, Roozendaal B, Nitsch RM, McGaugh JL, Hock C (2000) Acute cortisone administration impairs retrieval of longterm declarative memory in humans. Nat Neurosci 3:313-314

de Quervain DJ, Aerni A, Roozendaal B (2007) Preventive effect of beta-adrenoceptor blockade on glucocorticoid-induced memory retrieval deficits. Am J Psychiatry 164:967-969

Debiec J, Ledoux JE (2004) Disruption of reconsolidation but not consolidation of auditory fear conditioning by noradrenergic blockade in the amygdala. Neuroscience 129:267-272

Debiec J, Ledoux JE (2006) Contribution of noradrenergic transmission to memory reconsolidation in animals and humans: implications for PTSD. Biol Psychiatry 59:14S-15S

Diergaarde L, Schoffelmeer ANM, De Vries TJ (2008) Pharmacological manipulation of memory reconsolidation: towards a novel treatment of pathogenic memories. Eur J Pharmacol 585:453-457

Glannon W (2006) Psychopharmacology and memory. J Med Ethics 32:74-78

Greenberg DL, Rice HJ, Cooper JJ, Cabeza R, Rubin DC, LaBar KS (2005) Co-activation of the amygdala, hippocampus and inferior frontal gyrus during autobiographical memory retrieval. Neuropsychologia 43:659-674

Het S, Wolf OT (2007) Mood changes in response to psychosocial stress in healthy young women: effects of pretreatment with cortisol. Behav Neurosci 121:11-20

Het S, Ramlow G, Wolf OT (2005) A meta-analytic review of the effects of acute cortisol administration on human memory. Psychoneuroendocrinology 30:771-784

Kuhlmann S, Wolf OT (2005) Cortisol and memory retrieval in women: influence of menstrual cycle and oral contraceptives. Psychopharmacology (Berl) 183:65-71

Kuhlmann S, Wolf OT (2006) Arousal and cortisol interact in modulating memory consolidation in healthy young men. Behav Neurosci 120:217-223

Kuhlmann S, Kirschbaum C, Wolf OT (2005a) Effects of oral cortisol treatment in healthy young women on memory retrieval of negative and neutral words. Neurobiol Learn Mem 83:158-162

Kuhlmann S, Piel M, Wolf OT (2005b) Impaired memory retrieval after psychosocial stress in healthy young men. J Neurosci 25:2977-2982

Lupien SJ, McEwen BS (1997) The acute effects of corticosteroids on cognition: integration of animal and human model studies. Brain Res Rev 24:1-27

Maroun M, Akirav I (2007) Arousal and stress effects on consolidation and reconsolidation of recognition memory. Neuropsychopharmacology 33:394-405

McCleery JM, Harvey AG (2004) Integration of psychological and biological approaches to trauma memory: implications for pharmacological prevention of PTSD. J Trauma Stress 17:485-496

Miller MM, Altemus M, Debiec J, LeDoux JE, Phelps EA (2004) Propranolol impairs reconsolidation of conditioned fear in humans. Soc Neurosci Abs 208.2

Nater UM, La Marca R, Florin L, Moses A, Langhans W, Koller MM, Ehlert U (2006) Stress-induced changes in human salivary alphaamylase activity - associations with adrenergic activity. Psychoneuroendocrinology 31:49-58

O'Carroll RE, Drysdale E, Cahill L, Shajahan P, Ebmeier KP (1999) Stimulation of the noradrenergic system enhances and blockade reduces memory for emotional material in man. Psychol Med 29:1083-1088 
Palomba D, Angrilli A, Mini A (1997) Visual evoked potentials, heart rate responses and memory to emotional pictorial stimuli. Int $\mathbf{J}$ Psychophysiol 27:55-67

Pitman RK, Orr SP, Forgue DF, Dejong JB, Claiborn JM (1987) Psychophysiological assessment of posttraumatic-stress-disorder imagery in Vietnam combat veterans. Arch Gen Psychiatry 44:970-975

Pitman RK, Sanders KM, Zusman RM, Healy AR, Cheema F, Lasko NB, Cahill L, Orr SP (2002) Pilot study of secondary prevention of posttraumatic stress disorder with propranolol. Biol Psychiatry 51:189-192

Przybyslawski J, Roullet P, Sara SJ (1999) Attenuation of emotional and nonemotional memories after their reactivation: role of beta adrenergic receptors. J Neurosci 19:6623-6628

Rohleder N, Nater UM, Wolf JM, Ehlert U, Kirschbaum C (2004) Psychosocial stress-induced activation of salivary alpha-amylase: an indicator of sympathetic activity. Ann N Y Acad Sci 1032:258-263

Rohleder N, Wolf JM, Maldonado EF, Kirschbaum C (2006) The psychosocial stress-induced increase in salivary alpha-amylase is independent of saliva flow rate. Psychophysiology 43:645-652

Schelling G, Kilger E, Roozendaal B, de Quervain DJF, Briegel J, Dagge A, Rothenhausler HB, Krauseneck T, Nollert G, Kapfhammer HP (2004) Stress doses of hydrocortisone, traumatic memories, and symptoms of posttraumatic stress disorder in patients after cardiac surgery: a randomized study. Biol Psychiatry 55:627-633

Soravia LM, Heinrichs M, Aerni A, Maroni C, Schelling G, Ehlert U, Roozendaal B, de Quervain DJF (2006) Glucocorticoids reduce phobic fear in humans. Proc Natl Acad Sci U S A 103:5585-5590

Spielberger CD (1983) Manual for the state-trait anxiety inventory. Consulting Psychologists Press, Palo Alto, CA

Suzuki A, Josselyn SA, Frankland PW, Masushige S, Silva AJ, Kida S (2004) Memory reconsolidation and extinction have distinct temporal and biochemical signatures. J Neurosci 24:4787-4795

Tollenaar MS, Elzinga BM, Spinhoven P, Everaerd WA (2008a) The effects of cortisol increase on long-term memory retrieval during and after acute psychosocial stress. Acta Psychol (Amst) $127: 542-552$
Tollenaar MS, Elzinga BM, Spinhoven P, Everaerd W (2008b) Longterm outcomes of memory retrieval under stress. Behav Neurosci 122:697-703

Tollenaar MS, Elzinga BM, Spinhoven P, Everaerd WA (2008c) Immediate and prolonged effects of cortisol, but not propranolol, on memory retrieval in healthy young men. Neurobiol Learn Mem. doi:10.1016/j.nlm.2008.08.002

Tronel S, Alberini CM (2007) Persistent disruption of a traumatic memory by postretrieval inactivation of glucocorticoid receptors in the amygdala. Biol Psychiatry 62:33-39

Vaiva G, Ducrocq F, Jezequel K, Averland B, Lestavel P, Brunet A, Marmar CR (2003) Immediate treatment with propranolol decreases posttraumatic stress disorder two months after trauma. Biol Psychiatry 54:947-949

van der Does AJW (2002) BDI-II-NL Handleiding. De Nederlandse versie van de Beck Depression Inventory-second edition [BDI-II Dutch manual]. Swets and Zeitlinger, Lisse

van Stegeren A (2005) Beta-blockers in post-traumatic stress disorder: uses and controversies. Expert Rev Neurother 5:699-702

van Stegeren AH, Everaerd W, Cahill L, McGaugh JL, Gooren LJG (1998) Memory for emotional events: differential effects of centrally versus peripherally acting beta-blocking agents. Psychopharmacology 138:305-310

van Stegeren AH, Everaerd W, Gooren LJG (2002) The effect of betaadrenergic blockade after encoding on memory of an emotional event. Psychopharmacology 163:202-212

van Stegeren A, Rohleder N, Everaerd W, Wolf OT (2005) Salivary alpha amylase as marker for adrenergic activity during stress: effect of betablockade. Psychoneuroendocrinology 31:137-141

Wolf OT (2008) The influence of stress hormones on emotional memory: relevance for psychopathology. Acta Psychol (Amst) 127:513-531

Yang YL, Chao PK, Lu KT (2005) Systemic and intra-amygdala administration of glucocorticoid agonist and antagonist modulate extinction of conditioned fear. Neuropsychopharmacology 31:912-924 